\title{
Subjective assessment of right ventricle enlargement from computed tomography pulmonary angiography images
}

\author{
Markus Weininger
}

Received: 31 May 2011/Accepted: 9 June 2011/Published online: 18 June 2011

(C) Springer Science+Business Media, B.V. 2011

Driven by the evolution of technology Computed Tomography (CT) has become the methodology of choice to confirm or exclude the clinical suspicion of pulmonary artery embolism (PE) [1-6]. With its high temporal and spatial resolution, multi-detector CT has shown unique abilities in characterizing pulmonary embolisms with high sensitivity and specificity and is now serving as the sole reference standard for pulmonary embolism imaging in many institutions [7-9]. Most recent technological advancements, such as dual-energy imaging, further refine CT's capabilities by allowing the visualization of iodine distribution throughout the lung parenchyma [10-12].

Solidified by significant scientific data and research efforts in the diagnosis of pulmonary embolism, the important question no longer concerns demonstrating CT's clinical value in diagnosing pulmonary embolism, but optimizing the use in various clinical settings and patient categories. Based on results of investigative trials, such as Prospective Investigation of Pulmonary Embolism Diagnosis (PIOPED) II, diagnostic algorithms and clinical pathways have been

Editorial comment on the article of Kumamaru et al. (doi: 10.1007/s10554-011-9903-5).

M. Weininger $(\square)$

Department of Radiology and Radiological Science, Medical University of South Carolina, 25 Courtenay Drive, MSC 226, Charleston, SC 29425, USA

e-mail: mweininger1@yahoo.com proposed to formulate the appropriate indication of CT in the rule-out of PE [5, 13-15]. In a quest to evolve CT's competence beyond a pure as-is state interpretation into a tool for prognosis, applying the concept of evaluating the size and function of the right ventricle (RV) for risk assessment has been studied [16-18]. Initiated by the study of Reid et al., various studies compared right-ventricular measurements and ratios of right-ventricular diameters to left-ventricular (LV) diameters in a variety of image reconstructions, from true 4-chamber views to axial images [19-23]. Besides one-dimensional measurements, volumetric assessment of the right ventricle, although more timeconsuming, has been shown to be superior in the identification of high-risk patients [24].

In the current issue of the International Journal of Cardiovascular Imaging, Kumamaru et al. investigate a straightforward approach for the quantification of the size of the right ventricle. They hypothesized that a clear-cut, subjective visual assessment of rightventricular sizes on axial CT pulmonary angiography images is highly correlated with quantitative measurements. To validate their hypothesis, Kumamaru et al. evaluated the accuracy of their straightforward methodology using axial images and compared their findings to the prognostic accuracy of more traditional, quantitative RV/LV diameter ratios. Without using measurement tools, Kumamaru et al. had two readers retrospectively analyze axial CT images of 200 patients diagnosed with acute PE by CT pulmonary angiography to subjectively determine 
whether or not the maximum RV diameter was greater than that of the LV. Secondly, for objective measurements, all images were evaluated using a 3D post-processing workstation and $\mathrm{RV} / \mathrm{LV}$ diameter ratios were calculated on 4-chamber views and axial images. Clinical outcome data were included to allow for prognostic conclusions and all measurements were compared to each other.

As main findings, they discovered that among all interpretations for RV enlargement, the best agreement was between the two subjective assessments (91.5\%). Considering only patients with RV/LV diameter ratio $<0.93$ (calculated by 4-chamber method) and patients with RV/LV diameter ratio $>1.1$ (calculated by 4-chamber method), the two readers had perfect agreement. Correlation of the RV/ $\mathrm{LV}$ diameter ratio between 4-chamber (mean $1.07 \pm$ 0.26 ) and axial measurements (mean $1.10 \pm 0.27$ ) was high. With respect to the prediction of patient outcomes, they did not find a significant difference between subjective and objective assessments.

Based on their findings they concluded that a subjective assessment of RV enlargement is a simple and time-saving method to provide prognostic information in patients with acute pulmonary embolism, and more complex measurements of right-ventricular to left-ventricular diameter ratios may not be necessary. They further recommend that when the right ventricle subjectively appears larger than the left ventricle, this should be reported and considered for clinical risk stratification.

The current study is unique in the sense that it suggests a very pragmatic approach to enhance the value of image interpretation in patients with acute pulmonary embolism. It allows radiologist to offer clinicians precious additional information that is easily derived from CT images. In addition, Kumamaru et al. remind us that in a modern world, where we tend to rely only on latest generation postprocessing tools and have the tendency to only believe truly objective measured numbers, we should never neglect our subjective radiological judgment, experience and knowledge of image interpretation.

In summary, Kumamaru et al. convincingly demonstrate that relying on our radiological instincts is not outdated, but rather offers superior value, which put us in the center point facilitating clinical decision making.
Conflicts of interest None.

\section{References}

1. Remy-Jardin M, Remy J, Artaud D, Deschildre F, Duhamel A (1997) Peripheral pulmonary arteries: optimization of the spiral CT acquisition protocol. Radiology 204:157-163

2. Remy-Jardin M, Remy J, Wattinne L, Giraud F (1992) Central pulmonary thromboembolism: diagnosis with spiral volumetric CT with the single-breath-hold technique-comparison with pulmonary angiography. Radiology $185: 381-387$

3. Safriel Y, Zinn H (2002) CT pulmonary angiography in the detection of pulmonary emboli: a meta-analysis of sensitivities and specificities. Clin Imaging 26:101-105

4. Stein PD, Beemath A, Quinn DA et al (2007) Usefulness of multidetector spiral computed tomography according to age and gender for diagnosis of acute pulmonary embolism. Am J Cardiol 99:1303-1305

5. Stein PD, Fowler SE, Goodman LR et al (2006) Multidetector computed tomography for acute pulmonary embolism. N Engl J Med 354:2317-2327

6. Stein PD, Woodard PK, Weg JG et al (2007) Diagnostic pathways in acute pulmonary embolism: recommendations of the PIOPED II Investigators. Radiology 242:15-21

7. Eng J, Krishnan JA, Segal JB et al (2004) Accuracy of CT in the diagnosis of pulmonary embolism: a systematic literature review. AJR Am J Roentgenol 183:1819-1827

8. Hunsaker AR, Lu MT, Goldhaber SZ, Rybicki FJ (2010) Imaging in acute pulmonary embolism with special clinical scenarios. Circ Cardiovasc Imaging 3:491-500

9. Van Strijen MJ, De Monye W, Kieft GJ, Pattynama PM, Prins MH, Huisman MV (2005) Accuracy of singledetector spiral CT in the diagnosis of pulmonary embolism: a prospective multicenter cohort study of consecutive patients with abnormal perfusion scintigraphy. $\mathrm{J}$ Thromb Haemost 3:17-25

10. Fink C, Johnson TR, Michaely HJ et al (2008) Dual-energy CT angiography of the lung in patients with suspected pulmonary embolism: initial results. Rofo 180:879-883

11. Krissak R, Henzler T, Reichert M, Krauss B, Schoenberg SO, Fink C (2010) Enhanced visualization of lung vessels for diagnosis of pulmonary embolism using dual energy CT angiography. Invest Radiol 45:341-346

12. Thieme SF, Graute V, Nikolaou K, Maxien D, Reiser MF, Hacker M, Johnson TR (2010) Dual Energy CT lung perfusion imaging-Correlation with SPECT/CT. Eur J Radiol. doi:10.1016/j.ejrad.2010.11.037

13. Perrier A, Roy PM, Sanchez O et al (2005) Multidetectorrow computed tomography in suspected pulmonary embolism. N Engl J Med 352:1760-1768

14. van Belle A, Buller HR, Huisman MV et al (2006) Effectiveness of managing suspected pulmonary embolism using an algorithm combining clinical probability, D-dimer testing, and computed tomography. JAMA 295:172-179

15. Wells PS, Anderson DR, Rodger M et al (2001) Excluding pulmonary embolism at the bedside without diagnostic imaging: management of patients with suspected pulmonary embolism presenting to the emergency department by 
using a simple clinical model and d-dimer. Ann Intern Med 135:98-107

16. Goldhaber SZ, Elliott CG (2003) Acute pulmonary embolism: part II: risk stratification, treatment, and prevention. Circulation 108:2834-2838

17. Greyson CR (2011) Evaluation of right ventricular function. Curr Cardiol Rep 13:194-202

18. Ribeiro A, Lindmarker P, Juhlin-Dannfelt A, Johnsson H, Jorfeldt L (1997) Echocardiography Doppler in pulmonary embolism: right ventricular dysfunction as a predictor of mortality rate. Am Heart J 134:479-487

19. Araoz PA, Gotway MB, Trowbridge RL et al (2003) Helical CT pulmonary angiography predictors of in-hospital morbidity and mortality in patients with acute pulmonary embolism. J Thorac Imaging 18:207-216

20. Bankier AA, Janata K, Fleischmann D et al (1997) Severity assessment of acute pulmonary embolism with spiral CT: evaluation of two modified angiographic scores and comparison with clinical data. J Thorac Imaging 12:150-158
21. Chae EJ, Seo JB, Jang YM et al (2010) Dual-energy CT for assessment of the severity of acute pulmonary embolism: pulmonary perfusion defect score compared with CT angiographic obstruction score and right ventricular/left ventricular diameter ratio. AJR Am J Roentgenol 194:604-610

22. Ghuysen A, Ghaye B, Willems V et al (2005) Computed tomographic pulmonary angiography and prognostic significance in patients with acute pulmonary embolism. Thorax 60:956-961

23. van der Meer RW, Pattynama PM, van Strijen MJ et al (2005) Right ventricular dysfunction and pulmonary obstruction index at helical CT: prediction of clinical outcome during 3-month follow-up in patients with acute pulmonary embolism. Radiology 235:798-803

24. Henzler T, Krissak R, Reichert M, Sueselbeck T, Schoenberg SO, Fink C (2010) Volumetric analysis of pulmonary CTA for the assessment of right ventricular dysfunction in patients with acute pulmonary embolism. Acad Radiol 17:309-315 